\title{
Modelo educativo basado en el Tul Taekwondo para desarrollar valores en estudiantes de secundaria
}

\author{
Educational Model based on Tullow Tae Kwon Do to develop values in High School students \\ Eduardo Alfredo Espinoza Urbina ${ }^{1}$ \\ Juan Pablo Moreno Muro²
}

\section{Resumen}

El estudio tuvo como objetivo aplicar el Modelo del Tul Taekwondo que permita el desarrollo de valores en estudiantes de educación secundaria, por ser guías que orientan su conducta. El Taekwondo es la base del presente modelo ante el bajo nivel de desarrollo de valores, que es una de las causas de la disminución de sus aprendizajes y deserción escolar, contraviniendo lo estipulado en el Diseño Curricular Peruano. Siendo necesario revalorar el Área de Educación Física y la práctica del Tul Taekwondo, debiendo considerársele en el currículo escolar por favorecer la formación y conducción de las personas y el desarrollo de valores.

La metodología y el método fueron cuantitativo y experimental respectivamente. La población fue 319 estudiantes de la IEP "Ramón Espinoza Sierra" del departamento de Lambayeque y la muestra estuvo constituida por 60 estudiantes. Para la recopilación de datos, se utilizó la Ficha de Observación de Valores en la Actividad Diaria. Los datos se tabularon en el aplicativo SPSS, obteniéndose resultados descriptivos y las tablas correspondientes.

Los resultados demostraron que el Modelo causó efectos positivos en el desarrollo de valores, ya que evidenciaron diferencias positivas significativas entre el Pre test y Post test.

Palabras clave: modelo; desarrollo de valores; tul taekwondo o formas.

\section{Summary}

The study aimed to apply the Tullow Tae Kwon Do Model that allows the development of values in secondary school students, as they are guides that lead to good behavior. Tae Kwon Do is the basis of this model as an answer to the low level of values development, which is one of the causes of the decrease in their learning and dropping out of school, in contravention of what is stipulated in the Peruvian Curriculum Design.

\footnotetext{
1 Magister en Administración de Empresas. Correo Electrónico: eduardoespinoza10o7@hotmail.com; ORCID: https://orcid. org/oooo-0001-5868-5258

2 Doctor en Ciencias de la Educación. Docente de la Universidad César Vallejo. Correo Electrónico: paulmorenom@gmail.com; ORCID: https://orcid.org/ oooo-0002-5236-7520
}

Recibido: 14/01/2020 Aprobado: 10/03/2020 
Being necessary to reevaluate the Physical Education Area and the practice of Tullow Tae Kwon Do, and it should be considered in the school curriculum for promoting the training and management of people and the development of values.

The methodology and the method were quantitative and experimental respectively. The population was 319 students from the IEP "Ramón Espinoza Sierra" in the department of Lambayeque and the sample consisted of 60 students.

For data collection, the Observation Sheet of Values in Daily Activity was used. The data were tabulated in the SPSS application, obtaining descriptive results and the corresponding tables. The results demonstrated that the Model had positive effects in the development of values, the significant different evidences were manifested between the Pre Test and the Post Test.

Keywords: Model; values development; Tullow Tae Kwon Do or Forms.

\section{Introducción}

Una de las preocupaciones de nuestra sociedad es la lucha contra el bajo desarrollo de valores en las interrelaciones que se forjan entre los seres humanos; las conductas antisociales e inhumanas lastimosamente se han cimentado en la sociedad como algo común. Se carece o en algunos casos no se respetan los valores y, en consecuencia, se está viviendo en iguales condiciones que en aquellas etapas de la historia humana donde prevalecía la guerra, la violencia, la injusticia, etcétera.

Los valores son cualidades de las cosas, acciones, sentimientos e ideas, seleccionadas de manera libre, consciente o no por el ser humano y sirven para orientar sus decisiones y sentimientos en la satisfacción de determinadas necesidades (Bertussi, 1998, s.p). Sin embargo, el desarrollo de los valores se ha abandonado con el paso del tiempo evidenciándose que los seres humanos dejan de lado su asimilación y práctica valiosa, además ha cambiado la forma como los aprecian ante la influencia de nuevas tendencias sociales, costumbres y con otros aspectos como la utilidad y el bienestar que puedan generar. Es aquí donde la educación tiene entre sus obligaciones, el brindar un buen trato a los estudiantes, promoviendo un óptimo clima escolar a través del fomento de relaciones respetuosas, sin discriminación y no violentas, lo que se traducirá en mayores logros académicos y menores niveles de abandono escolar (UNESCO, 2013, p.26).

El Perú, viene atravesando una profunda crisis en la práctica de valores fundamentales como el respeto, responsabilidad, honestidad, justicia, solidaridad y la tolerancia, etcétera, agudizándose problemas como la discriminación, corrupción y la violencia social. Actualmente las formas de manifestación de violencia más comunes en las 


\section{EDUCACIÓN}

instituciones educativas según la plataforma SíseVe son de 7 tipos: sexual, con uso de armas, de forma física, robo, psicológica, por Internet y verbal (MINEDU, 2013, s.p.).

Concretamente en la institución educativa pública de nivel secundaria "Ramón Espinoza Sierra" del distrito lambayecano de Chiclayo, provincia de Chiclayo, que cuenta con una población caracterizada por un bajo nivel de desarrollo de valores, se ha observado la existencia de casos de violencia que se traduce en deserción escolar, bajo rendimiento académico e inseguridad, apreciándose estudiantes socialmente pasivos, con altos índices de ansiedad y que son poco receptivos, convirtiéndose en un verdadero reto para la comunidad educativa, el ejercitar y promover los valores a partir de una dinámica conjunta, predicando con el buen ejemplo (Pinto, 2016, p.271).

\section{Revisión de literatura}

Por la utilidad para el presente estudio, se seleccionaron las siguientes investigaciones. Leal (2005) manifestó que, es importante explorar cómo los docentes trabajan los valores, además de cambiar la forma cómo los estudiantes relacionan y moldean los valores. Riveros (2011) afirmó que es importante potenciar espacios que contribuyan al desarrollo de la autoconfianza y el respeto por los demás. Sintetizando que es posible hacerlo desde las actividades propias del Taekwondo, a fin de conocer los conflictos, emociones, conductas de los estudiantes y cómo manejarlas. Chávez (2011) señaló que es importante capacitar a los docentes sobre conocimientos y beneficios de la práctica del Taekwondo, el cual posee una filosofía cuyos principios son: cortesía, integridad, perseverancia, autocontrol y espíritu indomable que fomentan una sana convivencia.

Luego de esta revisión de estos antecedentes, se concluyó que los valores permiten formar una cultura de paz; sin embargo, éstos sufren una crisis en su desarrollo, perjudicando la buena convivencia y los aprendizajes de los estudiantes, haciéndose necesario proyectos con estrategias que integren a los miembros de la sociedad, puede ser a través del área de Educación Física porque ésta facilita espacios adecuados por medio de los cuales se obtendrán los objetivos de la educación (Vargas y Orozco, 2003, p.120), y específicamente con la práctica Tul Taekwondo, por su contenido filosófico y la práctica de técnicas poderosas que garantizan la superación personal, equilibrio emocional y una condición física saludable.

\section{Materiales y métodos}

El estudio se sustentó en el enfoque positivista, planteándose la hipótesis y variables evaluadas a partir de las preguntas generadas, las cuales a través del método y diseño escogido se midieron en un determinado contexto; posteriormente se analizaron los resultados obtenidos por medio de métodos estadísticos, llegando a una serie de conclusiones importantes (Hernández, Fernández y Baptista, 2014, p.4). 
La aplicación del paradigma cuantitativo se inició con la recolección de datos para su análisis, a fin de contestar las preguntas del estudio y probar la hipótesis planteada. El método seleccionado fue experimental y el diseño, cuasi experimental con aplicación de pre y post test, manipulando la variable independiente, modelo educativo, de manera deliberada para observar sus efectos sobre la variable dependiente, desarrollo de valores.

La selección y asignación de los estudiantes objeto del estudio a los grupos control y experimental no fue aleatorio, sino intencional ya que los sujetos de estudio fueron escogidos antes de la aplicación del modelo. Posteriormente se aplicó el tratamiento experimental modelo educativo basado en la práctica del Tul Taekwondo y luego se aplicó el post test, evaluándose la variable dependiente en los dos grupos de estudio. En cuanto a la finalidad, la investigación fue de tipo aplicada, porque aportó evidencias empíricas, al mostrar que, con la aplicación del modelo educativo basado en la práctica del Tul Taekwondo, se observaron ciertas mejoras en el desarrollo de valores.

Se aplicó el diseño cuasi experimental, que se representa de la siguiente manera:

\begin{tabular}{|c|c|c|c|}
\hline G.E.: & 01 & $X$ & 03 \\
\hline G.C.: & 02 & & 04 \\
\hline
\end{tabular}

La población estuvo conformada por 319 estudiantes del nivel de Educación Secundaria de la Institución Educativa Pública "Ramón Espinoza Sierra" del distrito de Chiclayo, provincia de Chiclayo, departamento de Lambayeque y la muestra escogida por conveniencia estuvo constituida por los 60 estudiantes del VI ciclo del nivel secundaria.

Al iniciar el estudio, se realizó el análisis documental, revisándose la ficha personal de los estudiantes, el registro de incidencias, el anecdotario del docente, así como los resultados de las entrevistas realizadas a los padres de familia por parte de la institución educativa. En cuanto al recojo de la información, se utilizó la Técnica de la Observación y el instrumento utilizado fue la Ficha de Observación de Valores en la Actividad Diaria- EVAF, para medir el nivel de desarrollo de los valores: respeto, responsabilidad, honestidad, justicia, solidaridad y tolerancia, que constó de 30 ítems y fue diseñada en Escala de Likert. La confianza sobre el instrumento es que su aplicación deberá arrojar siempre información consistente (Hernández, Fernández y 
Baptista, 2014, p. 200). El resultado de este coeficiente es de 0,897, razón por lo que se concluyó que el instrumento evaluado fue confiable.

\section{Resultados y discusión}

\section{Dimensión: Respeto}

Tabla 1: Estudiantes en porcentaje-Pre Test y Post Test Grupo Control y Experimental

\begin{tabular}{|l|l|l|l|l|}
\hline & \multicolumn{2}{|c|}{ Pre-test } & \multicolumn{2}{c|}{ Post-test } \\
\hline & \multicolumn{2}{|c|}{ Número de estudiantes en porcentaje } & \multicolumn{2}{c|}{ Número de estudiantes en porcentaje } \\
\hline & Grupo Control en \% & $\begin{array}{c}\text { Grupo Experimental } \\
\text { en } \%\end{array}$ & Grupo Control en \% & $\begin{array}{c}\text { Grupo Experimental } \\
\text { en \% }\end{array}$ \\
\hline Bajo & 47 & 60 & 47 & 27 \\
\hline Medio & 30 & 30 & 27 & 50 \\
\hline Alto & 20 & 10 & 23 & 23 \\
\hline Muy Alto & 3 & 0 & 3 & 0 \\
\hline TOTAL & 100 & 100 & 100 & 100 \\
\hline
\end{tabular}

Fuente: elaboración propia.

En la Tabla 1 de la dimensión: respeto, en el pre test, se encontraron 14 (47\%) y 18 (6o\%) estudiantes en el Nivel Bajo tanto del grupo control y experimental respectivamente y 9 (30\%) para ambos grupos en el Nivel Medio, estudiantes con dificultades para respetar decisiones tomadas por toda el aula y cumplir normas de conducta. En la misma dimensión en el Post test, se hallaron 8 (27\%) y 15 (50\%) estudiantes en el Nivel Medio en el grupo control y experimental y 7 (23\%) en el Nivel Alto para ambos grupos, pertenecientes a estudiantes que muestran mejoras al cumplir normas de conducta y respetar las decisiones tomadas por toda el aula, dando expresiones de valorar a los demás.

\section{Dimensión: Responsabilidad}

Tabla 2: Estudiantes en porcentaje- Pre Test y Post Test Grupo Control y Experimental

\begin{tabular}{|l|l|l|l|l|}
\hline & \multicolumn{2}{|c|}{ Pre-test } & \multicolumn{2}{c|}{ Post-test } \\
\hline & \multicolumn{2}{|c|}{ Número de estudiantes en porcentaje } & \multicolumn{2}{c|}{ Número de estudiantes en porcentaje } \\
\hline & Grupo Control en \% & Grupo Experimental en \% & Grupo Control en \% & Grupo Experimental en \% \\
\hline Bajo & 53 & 60 & 53 & 27 \\
\hline Medio & 30 & 33 & 27 & 47 \\
\hline Alto & 17 & 7 & 20 & 23 \\
\hline Muy Alto & 0 & 0 & 0 & 3 \\
\hline
\end{tabular}




\begin{tabular}{|l|l|l|l|l|}
\hline TOTAL & 100 & 100 & 100 & 100 \\
\hline \multicolumn{4}{|c|}{ Fuente: elaboración propia. } \\
\hline
\end{tabular}

En la Tabla 2 de la dimensión: responsabilidad, en el pre test, se hallaron 16 (53\%) y 18 (60\%) estudiantes en el nivel Bajo y en el Nivel Medio 9 (30\%) y 10 (33\%) del grupo control y experimental respectivamente, pertenecientes a estudiantes con dificultades para prestar atención en clase y que no aceptan las normas para trabajar en grupo y de juego. Así mismo en esta misma dimensión en el Post test, se hallaron $8(27 \%)$ y $14(47 \%)$ estudiantes en el Nivel Medio del grupo control y experimental y $6(20 \%)$ y 7 (23\%) en el Nivel Alto del grupo control y experimental respectivamente y $1(3 \%)$ en el Nivel Muy Alto del grupo experimental, cifras que ya indican cumplimiento en sus obligaciones como estudiantes e interés en tomar mejores decisiones.

\section{Dimensión: Honestidad}

Tabla 3. Estudiantes en porcentaje- Pre Test y Post Test Grupo Control y Experimental

\begin{tabular}{|c|c|c|c|c|}
\hline & & Pre-test & & st-test \\
\hline & Número d & tudiantes en porcentaje & Número de es & diantes en porcentaje \\
\hline & $\begin{array}{l}\text { Grupo Control } \\
\text { en } \%\end{array}$ & Grupo Experimental en $\%$ & Grupo Control en \% & Grupo Experimental en \% \\
\hline Bajo & 53 & 63 & 50 & 20 \\
\hline Medio & 30 & 30 & 33 & 47 \\
\hline Alto & 17 & 7 & 17 & 33 \\
\hline Muy Alto & 0 & 0 & 0 & 0 \\
\hline TOTAL & 100 & 100 & 100 & 100 \\
\hline
\end{tabular}

Fuente: elaboración propia.

En la Tabla 3 de la dimensión: honestidad, en el pres test se hallaron 16 (53\%) y $19(63 \%)$ estudiantes en el nivel bajo del grupo control y experimental y en el Nivel Medio 9 (30\%) para ambos grupos, cifras importantes referidas a estudiantes que se les hace difícil decir la verdad y que generan conflictos al no querer devolver lo que se les ha prestado en algún momento. Y en esta misma dimensión, en el Post test, se hallaron 10 (33\%) y 14 (47\%) estudiantes en el Nivel Medio del grupo control y experimental y en el Nivel Alto $5(17 \%)$ y 10 (33\%) respectivamente, cifras trascendentes 
referidas a estudiantes que han mejorado su práctica de la sinceridad y que asumen la obligación de devolver lo que no es suyo.

\section{Dimensión: Justicia}

Tabla No. 4: Estudiantes en porcentaje- Pre Test y Post Test Grupo Control y Experimental

\begin{tabular}{|l|l|l|l|l|}
\hline & \multicolumn{2}{|c|}{ Pre-Test } & \multicolumn{2}{c|}{ Post-Test } \\
\hline & \multicolumn{2}{|c|}{ Número de estudiantes en porcentaje } & \multicolumn{1}{c|}{ Número de estudiantes en porcentaje } \\
\hline & Grupo Control en \% & Grupo Experimental en \% & $\begin{array}{c}\text { Grupo Control } \\
\text { en \% }\end{array}$ & $\begin{array}{c}\text { Grupo Experimental } \\
\text { en \% }\end{array}$ \\
\hline Bajo & 57 & 60 & 57 & 17 \\
\hline Medio & 33 & 33 & 30 & 57 \\
\hline Alto & 10 & 7 & 13 & 23 \\
\hline Muy Alto & 0 & 0 & 0 & 3 \\
\hline TOTAL & 100 & 100 & 100 & 100 \\
\hline
\end{tabular}

En la Tabla 4 de la dimensión: justicia, en el pres Test, se hallaron 17 (57\%) y 18(60\%) estudiantes en el Nivel Bajo del grupo control y experimental y en el Nivel Medio 10 (33\%), similar cifra para los dos grupos y se refiere a estudiantes con problemas al momento de cumplir normas, no reconociendo las virtudes de sus compañeros. Mientras que, en la misma dimensión, en el Post test, se hallaron 9 (30\%) y 17(57\%) estudiantes en el Nivel Medio del grupo control y experimental y en el Nivel Alto 4 (13\%) y 7 (23\%) respectivamente y 1 (3\%) para el grupo experimental, cifras que dan indicios de respeto de reglas e inicio de reconocimiento de las virtudes de los demás.

\section{Dimensión: Solidaridad}

Tabla No. 5: Estudiantes en porcentajes- Pre Test y Post Test Grupo Control y Experimental

\begin{tabular}{|l|l|l|l|l|}
\hline & \multicolumn{2}{|c|}{ Pre-Test } & \multicolumn{2}{c|}{ Post-Test } \\
\hline & \multicolumn{2}{|c|}{ Número de estudiantes en porcentaje } & \multicolumn{2}{c|}{ Número de estudiantes en porcentaje } \\
\hline & Grupo Control en \% & Grupo Experimental en $\%$ & Grupo Control en $\%$ & Grupo Experimental en \% \\
\hline Bajo & 57 & 60 & 50 & 27 \\
\hline Medio & 30 & 33 & 37 & 50 \\
\hline Alto & 13 & 7 & 13 & 20 \\
\hline Muy Alto & 0 & 0 & 0 & 3 \\
\hline TOTAL & 100 & 100 & 100 & 100 \\
\hline
\end{tabular}


En la Tabla 5 de la dimensión: solidaridad, en el pre test, se hallaron 17 (57\%) y 18 (60\%) estudiantes en el Nivel Bajo y 9 (30\%) y 10 (33\%) en el Nivel Medio de los grupos control y experimental respectivamente, estudiantes con poco interés en defender a sus compañeros víctimas de violencia, y que no quieren enseñar a hacer deberes ni donar artículos que ellos ya no usan. Mientras que, en la misma dimensión, en el Post test, se hallaron 11 (37\%) y 15(50\%) estudiantes en el Nivel Medio del grupo control y experimental y en el Nivel Alto 4 (13\%) y 6 (20\%) respectivamente y 1 (3\%) en el Nivel Muy Alto perteneciente al grupo experimental, cifras que revelan mejoría en querer colaborar para conseguir algo favorable para todos, prestando la ayuda solicitada por algún compañero.

\section{Dimensión: Tolerancia}

Tabla 6.Estudiantes en porcentajes-Pre Test y Post Test Grupo Control y Experimental

\begin{tabular}{|l|l|l|l|l|}
\hline & \multicolumn{2}{|c|}{ Pre-Test } & \multicolumn{2}{c|}{ Post-Test } \\
\hline & \multicolumn{2}{|c|}{ Número de estudiantes en porcentaje } & \multicolumn{2}{c|}{ Número de estudiantes en porcentaje } \\
\hline $\begin{array}{c}\text { Grupo Control } \\
\text { en } \%\end{array}$ & Grupo Experimental en \% & Grupo Control en \% & $\begin{array}{c}\text { Grupo Experimental } \\
\text { en } \%\end{array}$ \\
\hline Bajo & 50 & 50 & 50 & 27 \\
\hline Medio & 40 & 47 & 37 & 53 \\
\hline Alto & 10 & 3 & 13 & 17 \\
\hline Muy Alto & 0 & 0 & 0 & 3 \\
\hline TOTAL & 100 & 100 & 100 & 100 \\
\hline
\end{tabular}

Fuente: elaboración propia.

En la Tabla 6 de la dimensión: tolerancia en el pres test, encontramos $15(50 \%)$ y 15 (50\%) estudiantes en el Nivel Bajo para ambos grupos y 12 (40\%) y 14 (47\%) en el Nivel Medio de los grupos control y experimental respectivamente, cantidades importantes correspondientes a estudiantes con dificultades para cumplir las normas de convivencia, que no promueven el dialogo y la tolerancia y consecuentemente no comparten con sus compañeros. Mientras que, en esta misma dimensión, en el Post test, se hallaron 11 (37\%) y 16(53\%) estudiantes en el Nivel Medio del grupo control y experimental y en el Nivel Alto $4(13 \%)$ y 5 (17\%) respectivamente y 1(3\%) del Nivel Muy Alto del grupo experimental que respetan diferencias de cultura, religión, maneras de ser y diversidad sexual.

En la dimensión: respeto, comparando los resultados del pre y post test, se verificó que de $18(60 \%)$ estudiantes del grupo experimental en el Nivel Bajo disminuyeron a $8(15 \%)$, en el Nivel Medio de 9 (30\%) pasaron a ser 15 (50\%) y en el Nivel Alto de 3 $(10 \%)$ a 7 (23\%), estudiantes con cambios positivos relacionados con respetar las decisiones tomadas entre compañeros de clase, notándose que la comunicación abierta 


\section{EDUCACIÓN}

es muy importante para tomar decisiones beneficiosas para todos, además se percibieron mejoras en el cuidado de los bienes propios del aula y la utilización correcta de los mismos; concordando con Riveros (2011), quien concluyó que se debe potenciar espacios que contribuyan al desarrollo de habilidades relacionadas con la autoconfianza, el respeto por los demás, el compañerismo, etcétera., afirmando que la práctica del Taekwondo por su filosofía permite conocer las emociones del estudiante; confirmándose lo revisado por Dewey (1859), que considera a la escuela parte de la sociedad, donde el aprendizaje se basa en la participación activa del estudiante, pero que necesita la guía del docente, más aún si se trata de la creación de su propia escala de valores (Santrock, 2006, p.5).

En la dimensión: responsabilidad, luego del post test, se comprobó que los estudiantes del grupo experimental en el Nivel Bajo de 18 (6o\%) pasaron a ser 8 (27\%) en el Nivel Medio de 10 (33\%) a 14 (47\%) en el Nivel Alto de 2 (7\%) a 7 (23\%) y en el Nivel Muy Alto 1 (3\%) estudiante del grupo experimental; quienes mejoraron su nivel de atención durante clase sin necesidad de repetírseles las instrucciones, coincidiendo con Galván (2011) quien afirmó que con el Taekwondo los estudiantes logran comprender como organizar de forma exitosa sus actividades. Debiéndose considerar a Puícan (2017) que manifiesta que la enseñanza de la educación física, requiere de una revisión de sus procesos, ya que cada docente le impregna a su práctica diferentes estilos y planteamientos didácticos. Corroborando lo manifestando por Vygotsky, que ve al estudiante como protagonista de las interacciones sociales durante su vida escolar y fuera de ella, indicando la importancia que el docente enseñe en un contexto práctico y en ambientes sociales y culturales correctos. Donde el interés por el aprendizaje no debe centrase en lo que el estudiante ya conoce, sino en lo que aún le falta por aprender (Serrano y Troche 2003, p.107). Tomándose el aporte de Elkonin, que expresa que el juego es vital en la pre escolaridad y fundamental para que los estudiantes asuman normas éticas (Grenier, 2000, p.557).

En lo que respecta a la dimensión: honestidad, luego del post test se confirmó que, los estudiantes del grupo experimental en el Nivel Bajo de 19 (63\%), pasaron a ser 6 (20\%), en el Nivel Medio de 9 (30\%) a 14 (47\%) y en el Nivel Alto de 2 (7\%) a 10 (33\%), visualizándose cambios en lo estudiantes que muestran mejoras en cuanto al manejo de la sinceridad y asumir consecuencias, aceptando cuando cometen errores y también dan muestras de voluntad de devolver lo que se les ha prestado. Coincidiendo con Aguirre (2016), quien creó una guía metodológica basada en cultura física, dando la importancia al Judo, deporte que transmite la importancia de los valores tanto en el hogar como en la escuela al igual que el Tul Taekwondo, contraponiéndose con Figueroa (2013) al explicar que a pesar que el deporte vincula a gran número de personas, sin embargo, en el Perú toda la atención se centra en el deporte profesional, apreciándose el desinterés del Estado por la producción de programas deportivos que aporten al mejoramiento social, ya que el deporte no debe depender de una sola disciplina. Desatendiendo lo propuesto por Piaget revela que, la educación moral es un 
proceso intervenido por el desarrollo de la inteligencia, a través de las interacciones y experiencias que provienen de contextos sociales, por tanto, será invalorable la ascendencia de la escuela y la familia como facilitadores del desarrollo del juicio moral de los estudiantes. Indicando que el desarrollo moral transita desde una moral guiada casi impuesta hasta que llega a una moral autónoma basada en vínculos interpersonales recíprocos y de apoyo (Grenier, 2000, p.554).

En la dimensión: justicia, posterior al post test, se evidenció que los estudiantes del grupo experimental en el Nivel Bajo de 18 (60\%), disminuyeron a 5 (17\%), en el Nivel Medio de 10 (33\%) aumentaron a 17 (57\%), en el Nivel Alto de 2 (7\%) a 7 (23\%) y en el Nivel Muy Alto surgió un 1 (3\%) estudiante, lo que muestra una mejora para cumplir las normas de clase y de juego y de querer contribuir con dar algunas soluciones a los conflictos; cambios que concuerdan con Sarco (2014) al señalar que el Karate Do, contiene enseñanzas con sentido social y valores culturales, con reglas muy similares a los del Taekwondo, por ser ambos artes marciales que promueven la sana convivencia; concordante también con la investigación de Puícan (2017) indica que, es importante que el docente examine el ambiente donde realiza sus clases y analice las cualidades, valores, limitaciones y dificultades de sus estudiantes, considerando las nuevas tendencias y exigencias de la educación física, de la educación y de la sociedad. Resultados que coinciden con La Teoría Ecológica de Bronfenbrenner, que indica que los estudiantes, no sólo se desarrollan a partir de su genética o la educación, sino que hay otros elementos determinantes como la escuela, el vecindario, la cultura, etcétera.

En la dimensión: solidaridad, luego del post test, se verificó que los estudiantes del grupo experimental en el Nivel Bajo de 18 (60\%), disminuyeron a 8 (27\%), en el Nivel Medio de 10 (33\%) aumentó 15 (50\%), en el Nivel Alto de 2 (7\%) pasó a 6 (20\%) y en el Nivel Muy Alto apareció 1 (3\%) estudiante del grupo experimental, cambios en estudiantes que están considerando defender a sus compañeros cuando éstos son víctimas de violencia, que también los escuchan y apoyan y están tomando conciencia de las necesidades de los demás, concordando con Tarazona (2017) quien manifiesta que, la escuela es quien debe educar a fin de cambiar la formar de pensar de los estudiantes a través de los valores, promoviendo en ellos la reflexión y el análisis sobre sus decisiones personales y sociales; y con lo investigado con Serra (2008) quien indicó que el docente encargado del área de Educación Física, es la persona más respetada socialmente para todos los estudiantes que recién se inician en este proceso de aprendizaje, asemejándose con lo expresado por Dewey que considera que los estudiantes deben ser estimulados y desarrollarse a través de la guía del docente por medio de un aprendizajes significativos (Santrock, 2006, p.5). Procurando lograr lo indicado en la Teoría de la Clarificación de los Valores de Raths (1967), nos centra en el conocimiento de los valores, que sugiere que sean pensados y escogidos por cada uno de los estudiantes y que el docente debe limitarse a garantizar su elección (Mesa, 2004, p.5). 
En la Dimensión: tolerancia, luego del post test, se verificó que los estudiantes del grupo experimental en el Nivel Bajo de 15 (50\%), disminuyeron a 8 (27\%), en el Nivel Medio de 14 (47\%) subió a 16 (53\%), en el Nivel Alto de 1 (3\%) a 5 (17\%) y el Nivel Muy Alto surgió 1 (3\%) estudiante, lo que indica que disminuyeron sus dificultades para cumplir las normas de convivencia, además de compartir con sus compañeros sin discriminarlos, demostrando empatía y manteniendo un nivel comunicativo muy importante, manifestándose en aprender y valorar todo lo que sabe cada uno de ellos, respetando ideas, creencias o prácticas de los demás, lo que concuerda con Dardón que señaló la relación inversa que existe entre la agresividad y el Taekwondo, al indicar cómo logran manejar el nivel de agresividad los deportistas que practican este deporte, debiendo considerarse lo indicado por Leal (2005), quien descubrió que es primordial elaborar estrategias que integren a los docentes y apoderados por ser actores claves para que los estudiantes formen sus valores; coincidiendo con lo expuesto con la Teoría de Bandura (1977) quien manifiesta que, los valores se aprenden a través del reforzamiento positivo o negativo que se hace de su conducta y que el comportamiento depende del control ejercido por el entorno social. Los procesos cognitivos, vicarios y autoreflexivos son determinantes en el funcionamiento psicosocial de las personas, donde el pensamiento humano es valioso para la comprensión del entorno (Vielma Vielma y Salas, 2000, p. 34).

\section{Conclusiones}

Se aplicó la ficha de observación de Valores en la Actividad Diaria-EVAF, para diagnosticar el nivel de desarrollo de valores en los estudiantes del VI ciclo del nivel de educación secundaria de la Institución Educativa "Ramón Espinoza Sierra" de la ciudad de Chiclayo.

La revisión teórica realizada permitió concluir que cualquier propuesta para el desarrollo de valores, debe basarse en Teorías como la de Piaget, por la importancia que le da a la división del desarrollo cognitivo en etapas, pero sin la rigidez que éste propone al determinar el inicio de las mismas; así como en las Teorías Constructivistas que sostienen que los estudiantes deben participar activamente en su proceso de aprendizaje tomando siempre sus aprendizajes previos, los cuales se modificarán a través de la adquisición de otros nuevos conocimientos.

Los fundamentos teóricos revisados y apropiados dieron pase al diseño del modelo educativo basado en la práctica del Tul Taekwondo, que permitió desarrollar valores en estudiantes del VI ciclo del nivel de educación secundaria de la Institución Educativa "Ramón Espinoza Sierra" de la ciudad de Chiclayo, en concordancia con las competencias que propone el Currículo Nacional Educativo.

Se aplicó el modelo educativo basado en la práctica del Tul Taekwondo para desarrollar valores en estudiantes de educación secundaria y los resultados del post test, 
demostraron que su aplicación causó efectos positivos, mostrando alta correlación entre las variables investigadas, validando el modelo.

\section{Agradecimiento}

Esta publicación obtuvo el financiamiento de: El Fondo de Asistencia Internacional de los Estudiantes y Académicos Noruegos (SAIH).

\section{Lista de referencia}

Aguirre, G. V. (2016). Las técnicas básicas del judo y la práctica de valores deportivos en los estudiantes de educación básica de la escuela Mariscal Sucre (Tesis de maestría). Universidad de Ambato. Ecuador.

Bandura, A. (1977). Self-efficacy: toward a unifying theory of behavioral change, Psychological review.

Bernal, C., A. (2010). Metodología de la investigación. Administración, economía, humanidades y ciencias sociales. (3a ed.). Colombia: Prentice Hall.

Bertussi, G. T. (1998). Los valores y la moral en la escuela. Educação y Sociedade.

Chávez, L. (2011). Programa Escolar de Capacitación en Taekwondo para los Docentes de Educación Física del Municipio Mara. (Tesis de pregrado). Universidad de Zulia. Venezuela.

Dardón, S. M. (2014). Nivel de agresividad en deportistas que practican Taekwondo", realizado en la Asociación de Taekwondo de Quetzaltenango. (Tesis de pregrado). Universidad. Rafael Saldívar. Guatemala.

Figueroa, F. M. (2013). La producción de programas deportivos en el canal del estado. El caso de "Planeta Deporte" período 1998 - 2003. (Tesis de pregrado). Universidad Nacional Mayor de San Marcos. Perú.

Galván, C. J. (2011). El Taekwondo como estrategia para el equilibrio emocional y actitudinal. (Tesis de pregrado). Universidad Pedagógica Nacional. Colombia.

Grenier, M. E. (2000). La formación de valores en la edad preescolar. Ciencia y Sociedad.

Hernández, R., Fernández, C. y Baptista, P. (2014). Metodología de la Investigación. 6ta ed. México: Mc Graw Hill. 


\section{EDUCACIÓN}

Leal, J. C. (2005). Valores para Vivir: Un Programa Educativo Internacional. (Caracterización de las condiciones y los factores que facilitan su implementación en la escuela básica). (Tesis de pregrado). Universidad de Chile. Chile.

Martínez, J y Tocarruncho, J. (2018). Efectos de poomsaes de taekwondo como práctica corporal sobre las conductas disruptivas. (Tesis de Pregrado). Universidad Libre.

Mesa, J. (2004). Tendencias actuales en la educación moral, Revista Latinoamericana de Ciencias Sociales, Niñez y Juventud. 2 (1).

Pinto, R. (2016). La importancia de promover los valores del hogar hacia las escuelas primarias. Revista Ra Ximhai, 12 (3), 271-283.

Riveros, F. (2011). Influencia de la práctica de Taekwondo en el bienestar de un grupo de niños de un taller vecinal. (Tesis de pregrado). Universidad Academia de Humanismo Cristiano. Chile.

Santrock, J. W (2006). Psicología de la Educación. Colombia: McGraw Hill.

Sarco, P. J. (2014). La práctica del Karate-Do y su influencia en el desarrollo psicomotriz y conductual en niños y niñas de 8 a 10 años de edad. (Tesis de pregrado). Universidad de Guayaquil. Ecuador.

Serra, J. (2008). Factores que influencian la práctica de la actividad física en la población adolescente de la provincia de Huesca. (Tesis Doctoral). Universidad de Zaragoza.

Serrano, M. y Troche, P. (2003). Teorías psicológicas de la educación. ( $3^{a}$ ed,). México: Universidad Autónoma del Estado de México.

Tarazona, O.M. (2017). Formación en valores morales y convivencia escolar en los estudiantes de primer grado de primaria de la institución educativa $N^{\circ}$. 21544. La Villa - Sayán - 2015. (Tesis de Maestría). Universidad César Vallejo. Perú.

UNESCO (2013). Situación Educativa de América Latina y el Caribe: Hacia la educación de calidad para todos al 2015. Santiago: Salesianos Impresores S.A.

Vargas, P. y Orozco R. (2003). La importancia de la Educación Física en el currículo escolar. InterSedes.

Vielma Vielma, E. Salas, M.L. (200o). Aportes de las teorías de Vygotsky, Piaget, Bandura y Bruner. Paralelismo en sus posiciones en relación con el desarrollo. Revista Educare. 3 (9), 30-37. 